\title{
Comparação de métodos in vitro para a quantificação da digestibilidade da matéria seca e da fibra em detergente neutro de forragens e concentrados
}

[Comparison of in vitro methods to quantify the dry matter and neutral detergent fiber digestibility of forages and concentrates]

\author{
T.E. Silva ${ }^{1}$, E. Detmann ${ }^{1}$, L.F. Camacho ${ }^{1}$, E.O.S. Saliba ${ }^{2}$, M.N.N. Palma ${ }^{1}$, S.C. Valadares Filho ${ }^{1}$
}

1Universidade Federal de Viçosa - Viçosa, MG

${ }^{2}$ Escola de Veterinária - Universidade Federal de Minas Gerais - Belo Horizonte, MG

\begin{abstract}
RESUMO
Objetivou-se comparar a digestibilidade in vitro da matéria seca (DIVMS) e da fibra em detergente neutro (DIVFDN) de forragens e concentrados obtidos por intermédio das incubadoras Daisy ${ }^{\text {II }}$ e TE-150, combinadas com filter bags tipo F57 (Ankon ${ }^{\circledR}$ ) ou tecido não-tecido (TNT, $100 \mathrm{~g} / \mathrm{m}^{2}$ ), em relação aos valores obtidos utilizando-se o método de Tilley e Terry (1963). Foram utilizadas 25 amostras de alimentos concentrados e 25 de forragens. Não houve diferenças entre filter bags $(\mathrm{P}>0,39)$ para a DIVMS de forragens. Maiores valores de DIVMS $(\mathrm{P}<0,01)$ foram verificados para a incubadora TE-150 em relação à Daisy ${ }^{\mathrm{II}}$. As estimativas de DIVMS obtidas com incubadoras e filter bags foram superiores $(\mathrm{P}<0,01)$ àquelas obtidas com o método Tilley e Terry (1963). Observaram-se maiores valores de DIVFDN de forragens $(\mathrm{P}<0,01)$ com filter bags $\mathrm{F} 57$ em relação ao TNT e com a incubadora TE-150 em relação à Daisy ${ }^{\text {II }}$. Todos os valores de DIVFDN obtidos com incubadoras e filter bags foram superiores $(\mathrm{P}<0,01)$ aos obtidos com o método Tilley e Terry (1963). Todos os métodos apresentaram-se positiva e fortemente correlacionados $(\mathrm{P}<0,01)$, tanto para DIVMS como para DIVFDN. As repetibilidades e variâncias entre amostras para DIVMS e DIVFDN foram similares entre os métodos. Portanto, conclui-se que, em se tratando de avaliações comparativas entre alimentos, todos os métodos avaliados possuem capacidade similar de discriminação.
\end{abstract}

Palavras-chave: Daisy ${ }^{\text {II }}$, filter bags, repetibilidade, TE-150

\begin{abstract}
The objective was to compare in vitro digestibility of dry matter (IVDMD) and neutral detergent fiber (IVNDFD) of forages and concentrates obtained using the incubators Daisy ${ }^{I I}$ and TE-150, and filter bags F57 $\left(\right.$ Ankon $\left.{ }^{\circledR}\right)$ or non-woven textile $\left(N W T-100 \mathrm{~g} / \mathrm{m}^{2}\right)$ in comparison with the values obtained using the Tilley and Terry (1963) method. Twenty-five samples of concentrates and 25 samples of forages were used. There were no differences between filter bags $(P>0.39)$ for IVDMD of forages. Higher IVDMD $(P<0.01)$ were observed for TE-150 compared to Daisy ${ }^{I I}$. Estimates of IVDMD obtained with incubators and filter bags were higher $(P<0.01)$ than those obtained with the Tilley and Terry (1963) method. Higher IVNDFD $(P<0.01)$ were obtained with filter bags 557 regarding to NWT and TE-150 when compared to Daisy ${ }^{I I}$. All values of the IVNDFD obtained with incubators and filter bags were higher $(P<0.01)$ than those obtained with the Tilley and Terry (1963) method. Both IVDMD and IVNDFD variables were greater using TE-150 incubator than using Daisy ${ }^{I I}(P<0.04)$. Estimates obtained using $N W T$ filter bags were higher $(P<0.01)$ than $F 57$ filter bags. All methods were positively and strongly correlated $(P<0.01)$ to each other for IVDMD and IVNDFD. The repeatability and variances among samples for IVDMD and IVNDFD were similar among methods. In conclusion, all methods were similarly able to discriminate samples.
\end{abstract}

Keywords: Daisy ${ }^{I I}$, filter bags, repeatability, TE-150

Recebido em 15 de abril de 2016

Aceito em 3 de fevereiro de 2017

E-mail: tadeu.silva@ufv.br 


\section{INTRODUÇÃO}

Estimativas da digestibilidade in vitro da matéria seca (DIVMS) e da fibra em detergente neutro (DIVFDN) de alimentos ou dietas podem permitir aos nutricionistas predizer a digestibilidade in vivo dessas frações por intermédio de equações de regressão, devido às fortes correlações existentes entre os resultados de ambas as técnicas. Da mesma forma, programas de melhoramento de plantas forrageiras podem utilizar essas estimativas como critério de seleção, aumentando o poder de discernimento entre materiais de maior ou menor valor nutritivo (Tilley e Terry, 1963; Silva e Queiroz, 2002). Adicionalmente, ensaios de digestibilidade in vitro constituem alternativas mais baratas e menos laboriosas quando comparadas às estimativas obtidas por ensaios de digestão in vivo (Berchielli et al., 2006).

O método considerado padrão para estudos de digestibilidade in vitro é o proposto por Tilley e Terry (1963), o qual se baseia em duas etapas: a primeira, em que as amostras são incubadas individualmente em tubos contendo inóculo ruminal e solução tampão, e a segunda, em que o resíduo obtido após 48 horas de incubação é submetido à digestão ácida com pepsina (Tilley e Terry, 1963).

Recentemente, disponibilizou-se no mercado o fermentador artificial de rúmen denominado Daisy ${ }^{\text {II }}$ (Ankom Technology Co., Macedon, NY, EUA), com a vantagem de possibilitar a análise de várias amostras simultaneamente, reduzindose, assim, o labor empregado nas análises (Holden, 1999; Mabjeesh et al., 2000; Adesogan, 2002). No Brasil, a indústria nacional produziu equipamento com características similares ao Daisy ${ }^{\text {II }}$ a incubadora TE-150 (Tecnal Equipamentos Científicos, Piracicaba, SP, Brasil).

Estudos comparando os valores de DIVMS obtidos pelo método do fermentador artificial Daisy ${ }^{\text {II }}$ em relação ao tradicional Tilley e Terry (1963) indicaram que eles produzem resultados similares (Holden, 1999; Mabjeesh et al., 2000; Santos et al., 2000). Entretanto, estudos contrastando os resultados produzidos pelo fermentador TE-150 em relação ao tradicional Tilley e Terry (1963) são escassos na literatura.
Adicionalmente, para o uso de fermentadores ou incubadoras, indica-se a incubação das amostras no interior de filter bags, destacando-se entre estes o F57 (Ankom Technology Co., Macedon, NY, EUA). Contudo, o custo elevado desse tipo de material pode gerar entrave à sua utilização, o que abre possibilidades para o uso de materiais alternativos de menor custo, como o tecido nãotecido (TNT; Casali et al., 2009; Valente et al., 2011a).

Dessa forma, objetivou-se comparar as estimativas de DIVMS e DIVFDN de forragens e concentrados obtidos por intermédio das incubadoras Daisy ${ }^{\text {II }}$ e TE-150, combinadas com diferentes tipos de filter bags (F57 e TNT), em relação aos valores encontrados utilizando-se o método de Tilley e Terry (1963), quanto à repetibilidade e à capacidade de discriminação entre amostras.

\section{MATERIAL E MÉTODOS}

O experimento foi conduzido no Laboratório de Nutrição Animal do Departamento de Zootecnia da Universidade Federal de Viçosa, Viçosa, MG.

Foram utilizadas 25 amostras de forragens e 25 de concentrados, perfazendo um total de 50 amostras. Para o grupo de forragens, foram avaliadas 16 gramíneas e nove leguminosas e para o grupo de concentrados, 15 energéticos e 10 proteicos. As amostras de forragens com alto teor de umidade foram secas em estufa com ventilação forçada $\left(55^{\circ} \mathrm{C}\right) \mathrm{e}$, em conjunto com as demais, foram processadas em moinho de facas com peneira de porosidade de $1 \mathrm{~mm}$. Posteriormente, todas as amostras foram analisadas quanto aos teores de matéria seca (MS; método INCT-CA G-003/1), proteína bruta (PB; método INCT-CA N-001/1) e fibra insolúvel em detergente neutro (FDN; método INCT-CA F-002/1), conforme métodos preconizados pelo Instituto Nacional de Ciência e Tecnologia em Ciência Animal (INCT-CA; Detmann et al., 2012; Tab. 1).

Foram avaliados cinco diferentes métodos para quantificação da DIVMS e da DIVFDN: método proposto por Tilley e Terry (1963) e métodos obtidos pela combinação de duas incubadoras (Daisy ${ }^{\text {II }}$ e TE-150) e dois tipos de filter bags (F57 e TNT, 100g/m²). 
Tabela 1. Estatísticas descritivas para os teores de proteína bruta e fibra em detergente neutro das forragens e dos concentrados utilizados nos ensaios de digestibilidade in vitro

\begin{tabular}{ccccc}
\hline \multirow{2}{*}{ Amostras } & \multicolumn{4}{c}{ Estatística } \\
\cline { 2 - 4 } & Mínimo & Máximo & Média & Desvio-padrão \\
\hline & \multicolumn{4}{c}{ Proteína bruta $(\mathrm{g} / \mathrm{kg}$ de matéria seca) } \\
\hline Forragens & 20,6 & 201,2 & 69,9 & 36,3 \\
Concentrados & 47,4 & 591,4 & 186,9 & 143,9 \\
\hline Forragens & 450,1 & 770,1 & 620,0 & 100,7 \\
Concentrados & 170,9 & 552,1 & 283,2 & 111,5 \\
\hline
\end{tabular}

Os métodos avaliados centraram-se em uma etapa de digestão em líquido ruminal, omitindose a etapa com utilização de pepsina preconizada por Tilley e Terry (1963). Para que as comparações entre métodos se restringissem aos componentes instrumentais (i.e., equipamento, filter bags, etc.), componentes físico-químicos similares foram utilizados (i.e., temperatura, solução tampão, proporção inóculo:tampão, etc.) para todos os métodos, os quais foram avaliados simultaneamente em três baterias consecutivas para cada grupo de amostra (forragens e concentrados).

O inóculo ruminal foi obtido de um bovino macho, não castrado, fistulado no rúmen. Todos os procedimentos de fistulação e manejo conduzidos com o animal doador foram aprovados pelo Comitê de Ética em Experimentação Animal da Universidade Federal de Viçosa (Ceuap, protocolo número 044/2016). A dieta do animal doador foi composta por canade-açúcar e concentrado comercial com $22 \%$ de $\mathrm{PB}$, sendo a relação volumoso:concentrado de 80:20 com base na MS. O animal teve acesso irrestrito à água e à mistura mineral completa $(90 \mathrm{~g} / \mathrm{kg}$ de fósforo) e foi adaptado à dieta padrão por 14 dias anteriormente ao início das coletas.

A digesta ruminal (líquido e sólidos) foi coletada em vários pontos da interface líquido-sólido do ambiente ruminal imediatamente antes do início de cada bateria de incubação. Logo após a retirada, a digesta foi transferida para o interior de garrafas térmicas pré-aquecidas à temperatura de $39^{\circ} \mathrm{C}$. Posteriormente, a digesta ruminal foi acondicionada em liquidificador, o qual foi mantido em sala climatizada $\left(39^{\circ} \mathrm{C}\right)$, sendo homogeneizada por 30 segundos. Em seguida, o material homogeneizado foi filtrado em quatro camadas de gaze em frascos Erlenmeyer de 2L, também mantidos em sala climatizada.
Todo o processo de obtenção, transporte, homogeneização e filtragem do inóculo foi realizado cerca de 20 minutos antes do início das incubações.

Utilizou-se solução tampão proposta por McDougall (1948). Previamente à mistura da solução tampão com o inóculo ruminal, foram adicionados $5 \mathrm{~mL}$ de solução de ureia $(5,5 \mathrm{~g} / 100 \mathrm{~mL})$ para cada $300 \mathrm{~mL}$ da solução tampão de McDougall. Logo em seguida, o pH da solução foi reduzido para 6,80 por borbulhamento com $\mathrm{CO}_{2}$ por 15 a 20 minutos.

As avaliações in vitro para o método proposto por Tilley e Terry (1963) seguiram as recomendações descritas pelos autores para o primeiro estádio, excetuando-se $o$ fato de utilizarem-se frascos vedados e de serem retirados os gases por agulhas em vez de tubos de ensaio com válvulas de Bunsen.

Foram pesados em duplicatas, para cada amostra, aproximadamente $500 \mathrm{mg}$ de amostras secas ao ar, as quais foram acondicionadas em frascos tipo "penicilina" $(100 \mathrm{~mL})$. Posteriormente, foram adicionados $10 \mathrm{~mL}$ de inóculo ruminal e 40mL de solução tampão de McDougall (relação 1:4 inóculo e solução tampão). O espaço livre dos frascos foi imediatamente saturado com $\mathrm{CO}_{2}$, sendo estes fechados com tampas de borracha e lacres de alumínio. Os frascos foram acondicionados sobre mesa agitadora orbital (40rpm) em sala climatizada $\left(39^{\circ} \mathrm{C}\right)$. Para cada bateria, foram avaliados dois frascos "branco", ou seja, apenas com solução tampão e inóculo ruminal, a fim de se quantificar o material contaminante advindo do inóculo. O gás produzido nos frascos foi retirado com uma agulha, a cada três horas, nas primeiras 12 horas, e a cada seis horas, nas 36 horas restantes. 
Após as 48 horas de incubação, os frascos foram abertos, e o conteúdo transferido para cadinhos filtrantes (porosidade grossa), com auxílio de água destilada (temperatura superior a $90^{\circ} \mathrm{C}$ ). Em seguida, os cadinhos foram secos $\left(105^{\circ} \mathrm{C} / 24\right.$ horas) e pesados, obtendo-se o resíduo aparentemente não digerido da matéria seca.

Para a avaliação da DIVFDN, os cadinhos contendo o resíduo de incubação foram introduzidos no interior de coletores universais autoclaváveis $(120 \mathrm{~mL})$, adicionando-se $80 \mathrm{~mL}$ de solução de detergente neutro, produzida segundo Mertens (2002), com a omissão de sulfito de sódio, e $250 \mu \mathrm{L}$ de $\alpha$-amilase termoestável (Termamyl 2X). Os coletores com os cadinhos acondicionados no interior foram fechados com as respectivas tampas e autoclavados $\left(105^{\circ} \mathrm{C} / 1 \mathrm{~h}\right)$ segundo método descrito por Detmann et al. (2012) (INCT-CA - F-002/1). Após retirados da autoclave, os cadinhos foram novamente lavados com água destilada quente e, ao fim, com $30 \mathrm{~mL}$ de acetona, sendo, em seguida, secos $\left(105^{\circ} \mathrm{C} / 24\right.$ h) e pesados para obtenção do resíduo de FDN.

As avaliações in vitro utilizando as incubadoras TE-150 e Daisy ${ }^{\text {II }}$ seguiram as recomendações descritas no manual dessa última, excetuando-se o fato de a solução tampão utilizada ter sido a proposta por McDougall (1948).

Foram pesados, em quadruplicata, para cada filter bag, F57 ou TNT, 500mg de amostra. Os filter bags de TNT foram confeccionados para terem as medidas de superfície próximas aos filter bags F57, ou seja, $36 \mathrm{~cm}^{2}$, com dimensões de $4 \mathrm{~cm} \times 4,5 \mathrm{~cm}$. Após as amostras serem pesadas, os filter bags foram imediatamente selados a quente. Previamente aos procedimentos, todos os filter bags foram limpos e pesados conforme procedimentos sugeridos por Detmann et al. (2012).

As replicatas de cada amostra foram inseridas em dois jarros de cada incubadora, perfazendo o total de quatro jarros por incubadora contendo 25 filter bags cada (dois para avaliação de filter bags F57 e dois para avaliação de filter bags TNT). Para cada bateria, foi introduzido, em cada jarro, um filter bag "branco".

Em seguida, foram adicionados, em cada jarro, $400 \mathrm{~mL}$ de inóculo ruminal e $1600 \mathrm{~mL}$ de solução de McDougall (relação 1:4 inóculo e solução tampão). O espaço livre dos jarros foi imediatamente saturado com $\mathrm{CO}_{2}$, sendo estes, então, fechados e acondicionados no interior de sua respectiva incubadora, previamente aquecida a $39^{\circ} \mathrm{C}$.

Após 48 horas, os filter bags foram imediatamente lavados com água destilada quente (temperatura superior a $90^{\circ} \mathrm{C}$ ), exercendose leve pressão manual para retirada dos gases neles contidos. Após a lavagem, todos os filter bags foram secos $\left(105^{\circ} \mathrm{C} / 24 \mathrm{~h}\right)$ e pesados, obtendo-se o resíduo aparentemente não digerido da MS.

Para a avaliação da DIVFDN, os filter bags foram acondicionados em coletores universais autoclaváveis $(120 \mathrm{~mL})$, sendo adicionados $80 \mathrm{~mL}$ de solução de detergente neutro e $250 \mu \mathrm{L}$ de $\alpha$ amilase termoestável, como previamente descrito. Os coletores com os filter bags em seu interior foram autoclavados, como descrito anteriormente. Posteriormente, foram lavados com água destilada quente (temperatura superior a $90^{\circ} \mathrm{C}$ ) e acetona. Após a lavagem, os filter bags foram secos $\left(105^{\circ} \mathrm{C} / 24 \mathrm{~h}\right)$ e pesados para obtenção do resíduo de FDN.

Para todos os métodos, a DIVMS e a DIVFDN (D, $\mathrm{g} / \mathrm{kg}$ ) foram calculadas como:

$$
D=\frac{M-(R-B)}{M} \times 1000(1)
$$

em que: $\mathrm{M}=$ massa de MS ou FDN incubada (g); $\mathrm{R}=$ resíduo de MS ou FDN da incubação $(\mathrm{g})$; $\mathrm{B}=$ resíduo de MS ou FDN obtido nos frascos "branco" (g).

O período experimental teve duração de 12 dias, em que cada grupo de amostras foi analisado durante seis dias consecutivos, com realização de três baterias de incubação para cada grupo de amostras.

Os cinco tratamentos foram avaliados segundo esquema fatorial com tratamento adicional $2 \times 2$ +1 (Healy, 1956), composto pela combinação entre as duas incubadoras avaliadas (Daisy ${ }^{\text {II }} \mathrm{e}$ TE-150) e os dois tipos de filter bags (F57 e TNT), adicionando-se o método original proposto por Tilley e Terry (1963), com as devidas modificações previamente descritas. 
As análises de variância para os valores de DIVMS e DIVFDN foram realizadas separadamente para forragens e concentrados por intermédio do procedimento MIXED do SAS 9.4, conforme o modelo:

$Y_{i j k l}=\mu+A_{i}+B_{j}+M_{k}+\varepsilon_{i j k l}(2)$;

em que: $\mathrm{Y}_{\mathrm{ijkl}}=$ valor observado na alíquota $1 \mathrm{da}$ amostra i, na bateria j, utilizando-se o método k; $\mu=$ constante geral; $A_{i}=$ efeito da amostra $i$ (aleatório); $\mathrm{B}_{\mathrm{j}}=$ efeito da bateria de análise $\mathrm{j}$ (aleatório); $\mathrm{M}_{\mathrm{k}}=$ efeito do método $\mathrm{k}$ (fixo); e $\varepsilon_{\mathrm{ijkl}}=$ erro aleatório, não observável, pressuposto $\operatorname{NID}\left(0 ; \sigma^{2}\right)$.

A comparação entre os tratamentos baseados no uso de incubadoras e filter bags foi realizada por intermédio do teste $\mathrm{F}$ de Snedecor, seguindo o esquema fatorial $2 \times 2$. A comparação entre esses tratamentos e o método de Tilley e Terry (1963) foram conduzidos por intermédio do teste de Dunnett.

Após a primeira análise de variância, um segundo conjunto de análises de variância foi realizado, separadamente para forragens $\mathrm{e}$ concentrados, para mensuração da variação específica de cada método testado, conforme o modelo:

$Y_{i j k}=\mu+A_{i}+B_{j}+\varepsilon_{i j k}(3)$;

em que: $\mathrm{Y}_{\mathrm{ijk}}=$ valor observado na alíquota $\mathrm{k}$ da amostra $i$, na bateria $j ; \mu=$ constante geral; $A_{i}=$ efeito da amostra $\mathrm{i}$ (aleatório); $\mathrm{B}_{\mathrm{j}}=$ efeito da bateria de análise $\mathrm{j}$ (aleatório); e $\varepsilon_{\mathrm{ijk}}=$ erro aleatório, não observável, pressuposto NID (0; $\left.\sigma^{2}\right)$.

As estimativas da variância residual $\left(\hat{\sigma}_{\varepsilon}^{2}\right)$ e da variância entre amostras $\left(\hat{\sigma}_{a}^{2}\right)$ foram obtidas pelo método da máxima verossimilhança restrita.

A repetibilidade padronizada em função da média foi calculada como:

$r=\frac{\sqrt{\hat{\sigma}_{\varepsilon}^{2}}}{\bar{Y}} \times 100(4)$

em que: $r=$ repetibilidade padronizada em função da média $(\%) ; \hat{\sigma}_{\varepsilon}^{2}=$ variância residual; e $\bar{Y}=$ valor médio de digestibilidade.
A variância entre amostras foi também padronizada em função da média, a qual indica o poder de discriminação entre amostras de cada método. Ela é dada por:

$v=\frac{\sqrt{\hat{\sigma}_{a}^{2}}}{\bar{Y}} \times 100$

em que: $v=$ variância entre amostras padronizada em função da média $(\%) ; \hat{\sigma}_{a}^{2}=$ variância entre amostras; e $\bar{Y}=$ valor médio de digestibilidade.

A correlação linear de Pearson entre métodos foi estimada por intermédio da opção MANOVA do procedimento GLM do SAS 9.4, sendo esta ajustada para efeitos de bateria de análise. Adotou-se $\alpha=0,05$ para todos os procedimentos estatísticos.

\section{RESULTADOS}

Tanto para DIVMS como para DIVFDN, não se verificou variação entre baterias de análises $(\mathrm{P}>0,05)$. Esse fato reitera aspectos de rusticidade dos métodos utilizados.

Para a DIVMS de forragens, não se verificou efeito de interação entre incubadora e filter bags $(\mathrm{P}>0,12)$ ou diferenças entre filter bags $(\mathrm{P}>0,39)$; contudo, maiores valores $(\mathrm{P}<0,01)$ foram verificados para a incubadora TE-150 em relação à incubadora Daisy ${ }^{\text {II }}$ (Tab. 2). Para essa variável, as estimativas obtidas com incubadoras e filter bags foram superiores $(\mathrm{P}<0,01)$ àquelas obtidas com o método de Tilley e Terry (1963).

Verificou-se efeito de interação entre incubadora e filter bag $(\mathrm{P}<0,02)$ para a DIVFDN de forragens (Tab. 2). Contudo, o desdobramento desse efeito indicou que maiores valores $(\mathrm{P}<0,01)$ foram obtidos com filter bags $\mathrm{F} 57 \mathrm{em}$ relação ao TNT e com a incubadora TE-150 em relação à Daisy ${ }^{\text {II }}$. Todos os valores obtidos com incubadoras e filter bags foram superiores $(\mathrm{P}<0,01)$ aos obtidos com o método de Tilley e Terry (1963) (Tab. 2).

Não foi verificado efeito de interação entre incubadora e filter bag $(\mathrm{P}>0,20)$ para a DIVMS e a DIVFDN de concentrados (Tab. 2). Para ambas as variáveis, maiores estimativas foram obtidas com a incubadora TE-150 em relação à Daisy ${ }^{\text {II }}$ 
$(\mathrm{P}<0,04)$ e com filter bags TNT em relação ao F57 $(\mathrm{P}<0,01)$. Os métodos baseados no uso dos filter bags F57 produziram DIVMS em concentrados similares $(\mathrm{P}>0,34)$, enquanto o uso de TNT produziu resultados superiores $(\mathrm{P}<0,01)$ aos obtidos com o método de Tilley e Terry (1963). Todas as estimativas de DIVFDN de concentrados obtidas com o uso de incubadoras e filter bags foram superiores $(\mathrm{P}<0,01)$ às obtidas com o método de Tilley e Terry (1963).

Todos os métodos avaliados apresentaram-se positiva e fortemente correlacionados $(\mathrm{P}<0,01)$, tanto com relação à DIVMS como com relação à DIVFDN para forragens e concentrados (Tab. 3).

Tabela 2. Médias de mínimos quadrados e níveis descritivos de probabilidade para o erro tipo I em função dos diferentes contrastes avaliados (valor $\mathrm{P}$ ) dos valores de digestibilidade in vitro da matéria seca (DIVMS; g/kg) e da fibra em detergente neutro (DIVFDN; g/kg) para amostras de forragens e concentrados

\begin{tabular}{ccccc}
\hline & \multicolumn{2}{c}{ Forragens $^{2}$} & \multicolumn{2}{c}{ Concentrados $^{2}$} \\
\cline { 2 - 5 } Método $^{1}$ & DIVMS & DIVFDN & DIVMS & DIVFDN \\
\cline { 2 - 5 } Tilley e Terry (1963) & $525,4 \pm 20,8$ & $364,5 \pm 33,7$ & $851,2 \pm 25,5$ & $678,4 \pm 33,7$ \\
D/F57 & $572,6 \pm 20,8$ & $446,6 \pm 33,7$ & $845,4 \pm 25,5$ & $749,7 \pm 33,7$ \\
D/TNT & $575,0 \pm 20,8$ & $428,5 \pm 33,7$ & $863,5 \pm 25,5$ & $794,9 \pm 34,4$ \\
T/F57 & $605,6 \pm 20,8$ & $499,6 \pm 33,7$ & $854,6 \pm 25,5$ & $782,4 \pm 33,7$ \\
T/TNT & $597,4 \pm 20,8$ & $458,9 \pm 33,7$ & $865,9 \pm 25,5$ & $810,8 \pm 33,8$ \\
\hline Comparações & \multicolumn{4}{c}{ Valor P } \\
Esquema fatorial & $<0,001$ & $<0,001$ & 0,031 & 0,005 \\
Incubadora (I) & 0,395 & $<0,001$ & $<0,001$ & $<0,001$ \\
Filter bag (F) & 0,122 & 0,017 & 0,205 & 0,333 \\
I $\times$ F & $<0,001$ & $<0,001$ & $<0,001$ \\
\hline D/F57 & $<0,001$ & $<0,001$ & 0,348 & $<0,001$ \\
D/TNT & $<0,001$ & $<0,001$ & 0,001 & $<0,001$ \\
T/F57 & $<0,001$ & $<0,001$ & 0,787 & $<0,001$ \\
T/TNT & Comparações com o método de Tilley e Terry $(1963)$ & 0,004 & \\
\hline
\end{tabular}

${ }^{1} \mathrm{D}$, incubadora Daisy ${ }^{\mathrm{II}}$ T, incubadora TE-150; F57, filter bag F57; TNT, filter bag TNT.

${ }^{2}$ Média \pm erro-padrão da média.

Tabela 3. Estimativas da correlação linear de Pearson entre os valores digestibilidade in vitro da matéria seca (DIVMS) e da fibra em detergente neutro (DIVFDN) nas amostras de forragens e concentrados obtidos por diferentes métodos

\begin{tabular}{cccccc}
\hline & \multicolumn{5}{c}{ Métodos $^{\mathbf{2} \mathbf{3}}$} \\
\hline Métodos & TeT & D/F57 & D/TNT & T/F57 & T/TNT \\
\cline { 2 - 6 } T e T & - & DIVMS & 0,768 & 0,763 \\
D/F57 & 0,908 & 0,746 & 0,747 & 0,919 & 0,899 \\
D/TNT & 0,893 & - & 0,906 & 0,888 & 0,887 \\
T/F57 & 0,913 & 0,962 & - & - & 0,903 \\
T/TNT & 0,894 & 0,954 & 0,952 & 0,950 & - \\
\hline & & 0,950 & DIVFDN & 0,720 \\
T e T & - & 0,696 & 0,727 & 0,789 & 0,806 \\
D/F57 & 0,643 & - & 0,787 & 0,835 & 0,862 \\
D/TNT & 0,666 & 0,898 & - & 0,793 & 0,837 \\
T/F57 & 0,731 & 0,869 & 0,863 & - & - \\
T/TNT & 0,674 & 0,868 & 0,898 & 0,861 & .
\end{tabular}

${ }^{1}$ T e T, método de Tilley e Terry (1963); D, incubadora Daisy ${ }^{I I}$; T, incubadora TE-150; F57, filter bag F57; TNT, filter bag TNT. ${ }^{2}$ Valores acima e abaixo da diagonal principal correspondem a amostras de forragens e de concentrados, respectivamente. ${ }^{3}$ Todas as correlações foram significativas pelo teste t de Student $(\mathrm{P}<0,001)$. 
As repetibilidades para a DIVMS e a DIVFDN foram similares entre todos os métodos avaliados para as amostras de concentrados (Fig. 1). Contudo, verificou-se menor repetibilidade (maior valor de repetibilidade padronizada) para a DIVMS e a DIVFDN de forragens utilizandose o método de Tilley e Terry (1963).

As variâncias entre amostras de concentrados quanto à DIVMS foram similares entre métodos
(Fig. 1). No entanto, a variância entre amostras de forragens quanto à DIVFDN foi pouco superior para o método de Tilley e Terry (1963) em relação aos demais métodos. Por outro lado, a variância entre amostras de forragens quanto à DIVFDN foi similar entre os métodos, sendo esta, contudo, pouco superior para o método de Tilley e Terry (1963) para amostras de concentrados.

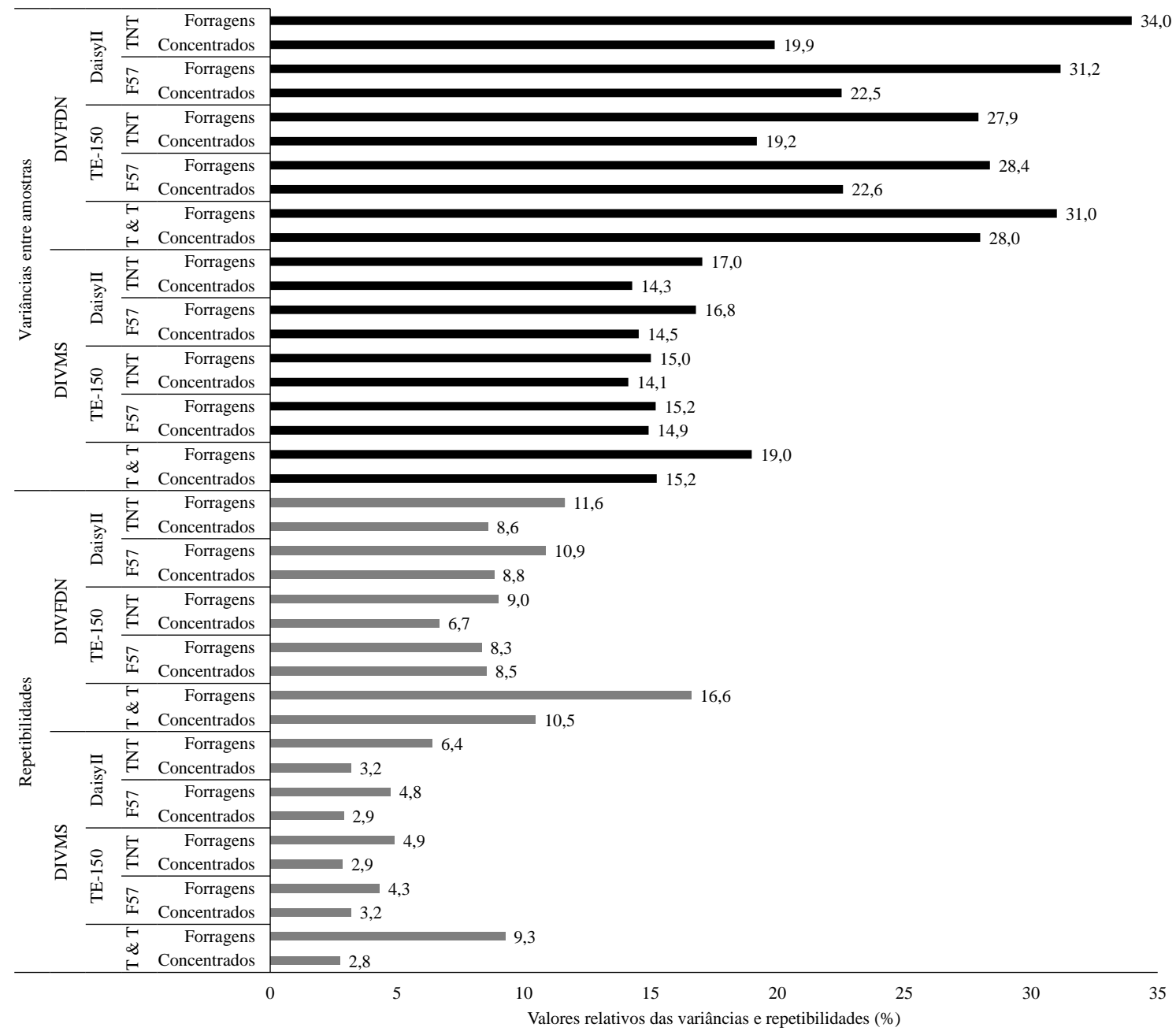

Figura 1. Repetibilidades e variâncias amostrais padronizadas das DIVMS e DIVFDN em função da média dos tratamentos para as amostras de forragens e concentrados.

\section{DISCUSSÃO}

De forma geral, com exceção da DIVMS de concentrados com o uso de filter bags F57 (Tab. 2), todas as avaliações conduzidas em incubadoras produziram resultados superiores ao observado com o método de Tilley e Terry (1963). Esse comportamento corrobora outros autores (Mabjeesh et al., 2000; Santos et al., 2000; Lopes et al., 2010; Duque et al., 2011), os quais, em média, obtiveram maiores estimativas de digestibilidade com o uso de incubadoras em 
comparação ao método de Tilley e Terry (1963). Essa superioridade das estimativas obtidas com incubadoras parece ser, ao menos parcialmente, atribuída à menor homogeneidade do ambiente de incubação em tubos comparados ao uso de jarros e filter bags. As amostras no método de Tilley e Terry (1963) são manejadas livres no interior dos frascos de incubação. Isso pode acarrretar adesão de partículas à superfície dos frascos em nível superior ao fluido (inóculo + tampão), prevenindo ou evitando sua digestão (Freitas et al., 2002).

Por outro lado, o uso de filter bags para avaliação in vitro ou in situ de amostras pode ocasionar diferenças entre os ambientes interno do filter bag e o meio externo (Ramin et al., 2013), o que parece advir de reduções nas taxas de troca entre ambientes quanto à saída de produtos de digestão e à entrada de novos substratos e microrganismos (Lindberg et al., 1984; Valente et al., 2011b). Essa característica estaria agregada aos fatores causadores das diferenças entre o uso do sistema convencional de Tilley e Terry (1963) e o uso de sistemas baseados em filter bags. Adicionalmente, deve se considerar que as amostras no método de Tilley e Terry (1963) são incubadas individualmente, ao passo que, com o uso de incubadoras, as amostras são avaliadas coletivamente. A incubação conjunta de amostras com diferentes composições químicas pode acarretar a ocorrência de efeitos associativos (Adesogan, 2002) e, desse modo, ampliar as estimativas de digestibilidade.

Por outro lado, em todas as avaliações, observaram-se diferenças entre as incubadoras avaliadas, com maiores estimativas, tanto de DIVMS como de DIVFDN, obtidas com a incubadora TE-150 (Tab. 2). De certa forma, essas diferenças parecem assumir caráter lógico, pois, embora sendo propostos para a mesma finalidade, os equipamentos avaliados possuem algumas características peculiares. Entre essas, destaca-se a rotação dos jarros contendo os filter bags no interior das incubadoras. Em mensurações realizadas durante as incubações, verificou-se rotação constante de $1 \mathrm{rpm}$ para a incubadora Daisy ${ }^{\mathrm{II}}$, ao passo que a incubadora TE-150 apresentou rotação média dos jarros de 10,82 $\pm 0,15 \mathrm{rpm}$ (média de quatro mensurações). A maior rotação da incubadora TE-150 pode ter influenciado o contato entre amostra e inóculo, ampliando, assim, as estimativas de digestibilidade.

Diferenças entre filter bags foram verificadas para três das quatro variáveis-resposta avaliadas (Tab. 2). Embora os filter bags de TNT e F57 tenham constituição física similar, ou seja, são produzidos em processo de não tecelagem, com a deposição não organizada das fibras (Casali et al., 2009), diferenças entre tecidos têm sido relatadas no tocante à superfície específica (Casali et al., 2009) e nas características de troca entre o interior do filter bag e o meio externo (Valente et al., 2011b).

As estimativas de digestibilidade in vitro podem ser enquadradas em dois principais objetivos: predição da digestibilidade in vivo e avaliação comparativa entre alimentos. No primeiro caso, as estimativas somente podem ser julgadas válidas caso haja possibilidade de avaliação simultânea in vitro e in vivo dos mesmos alimentos ou dietas. Assim, seria possível avaliar a exatidão do método aplicado, a qual reflete a discrepância (vício) entre os valores estimados in vitro e os valores assumidos como reais obtidos in vivo. Contudo, tais avaliações são escassas em condições brasileiras, não permitindo o julgamento adequado de métodos in vitro quanto à sua exatidão. Acresce-se a isso a ampla variação nos procedimentos e instrumentos utilizados para avaliação in vitro, o que caracteriza falta de padronização entre laboratórios.

Mesmo com a presença de vícios significativos, os métodos in vitro tendem a apresentar forte correlação com os resultados obtidos in vivo (Silva e Queiroz, 2002), o que permite estimar a digestibilidade por intermédio de equações de regressão (Åkerlind et al., 2011). A despeito das diferenças entre médias, todos os métodos aqui avaliados se mostraram fortemente correlacionados entre si (Tab. 3). Isso constitui indicativo de que todos podem apresentar o mesmo padrão de correlação com valores in vivo.

Para o caso de avaliação comparativa entre alimentos, duas características principais podem ser relevadas: precisão e capacidade de discriminação. A precisão reflete baixa participação do erro aleatório entre repetições, o que permite comparações mais confiáveis entre alimentos, reduzindo, principalmente a 
probabilidade de ocorrência do erro estatístico do tipo II. Neste trabalho, a precisão pode ser representada pela repetibilidade, pois ela está baseada na variância aleatória residual (Eq. 4). Verificou-se que os métodos baseados no uso de incubadoras apresentaram repetibilidade igual ou superior ao método de Tilley e Terry (1963) (Fig. 1). Esse comportamento parece refletir, como previamente discutido, a maior homogeneidade do contato amostra-fluido, além do menor número de procedimentos laboratoriais, principalmente pela não necessidade de transferência do resíduo pós-digestão para um recipiente filtrante.

A variância entre amostras (Eq. 5) reflete, para um mesmo grupo de amostras, a capacidade do método em discriminar elementos desse grupo com características de digestibilidade distintas. Nesse quesito, a despeito de pequenas discrepâncias, os métodos apresentaram comportamento similar.

Dessa forma, características secundárias, como custo, praticidade, capacidade operacional e acesso a equipamentos e suprimentos, devem ser consideradas como fatores preponderantes para a escolha do método a ser adotado.

\section{CONCLUSÕES}

Conclui-se que, em se tratando de avaliações comparativas entre alimentos, todos os métodos possuem capacidade similar de discriminação.

\section{AGRADECIMENTOS}

Ao CNPq, à Fapemig e ao INCT-Ciência Animal, pelo apoio financeiro.

\section{REFERÊNCIAS}

ADESOGAN, A.T. What are feeds worth? A critical evaluation of selected nutritive values methods. In: ANNUAL FLORIDA RUMINANT NUTRITION SYMPOSIUM, 13., 2002, Gainesville. Proceedings... Gainesville: University of Florida, 2002. p.33-47.

ÅKERLIND, M.; WEISBJERG, M.; ERIKSSON, T. et al. Feed analysis and digestion methods. In: VOLDEN, H. (Ed.). NorFor - the nordic feed evaluation system. Wageningen: Wageningen Academic Publishers, 2011. p.4154.
BERCHIELLI, T.T.; GARCIA, A.V.; OLIVEIRA, S.G. Principais técnicas de avaliação aplicadas em estudo de nutrição. In: BERCHIELLI, T.T.; VAZ PIRES, A.; OLIVEIRA, S.G. (Eds.). Nutrição de ruminantes. Jaboticabal: Funep, 2006. p.397421.

CASALI, A.O.; DETMANN, E.; VALADARES FILHO, S.C. et al. Estimação de teores de componentes fibrosos em alimentos para ruminantes em sacos de diferentes tecidos. Rev. Bras. Zootec., v.38, p.130-138, 2009.

DETMANN, E.; SOUZA, M.A.; VALADARES FILHO, S.C. et al. Métodos para análise de alimentos. Suprema: Visconde do Rio Branco, 2012. 214p.

DUQUE, A.C.A.; LOPES, F.C.F.; DORNELLAS, R.A.C. et al. Digestibilidade da matéria seca de alimentos volumosos e concentrados, determinada por diferentes procedimentos in vitro. Rev. Bras. Saúde Prod. Anim., v.12, p.680-690, 2011.

FREITAS, D.; BERCHIELLI, T.T.; SILVEIRA, R.N. et al. Produção fecal e fluxo duodenal de matéria seca e matéria orgânica estimados por meio de indicadores. Rev. Bras. Zootec., v.31, p.1521-1530, 2002.

HEALY, M.J.R. The analysys of a factorial experiment with additional treatments. J. Agric. Sci., v.47, p.205-206, 1956.

HOLDEN, L.A. Comparison of methods of in vitro dry matter digestibility for ten feeds. $J$. Dairy. Sci., v.82, p.1791-1794, 1999.

LINDGERG, J.E.; KASPERSSON, A.; CISZUK, P. Studies on $\mathrm{pH}$, number of protozoa and microbial ATP concentrations in rumenincubated nylon bags with different pore sizes. $J$. Agric. Sci., v.102, p.501-504, 1984.

LOPES, F.C.F.; DORNELAS, R.A.C.; PORTUGAL, J.A.B. et al. Digestibilidade da matéria seca de silagens de milho e de suplementos concentrados determinada por procedimentos in vitro. Arq. Bras. Med. Vet. Zootec., v.62, p.1167-1173, 2010.

MABJEESH, S.J.; COHEN, M.; ARIELI, A. In vitro methods for measuring the dry matter digestibility of ruminant feedstuffs: comparison of methods and inoculum source. J. Dairy Sci., v.83, p.2289-2294, 2000. 
McDOUGALL, E.I. The composition and output of sheep's saliva. Biochem. J., v.43, p.99-109, 1948.

MERTENS, D.R. Gravimetric determination of amylase treated neutral detergent fiber in feeds with refluxing in beaker or crucibles: collaborative study. J. Assoc. Off. Anal. Chem. Intern., v.85, p.1217-1240, 2002.

RAMIN, M.; KRIZSAN, S.J.; JANČÍK, F.; HUHTANEN, P. Measurements of methane emissions from feed samples in filter bags or dispersed in the medium in an in vitro gas production system. J. Dairy Sci., v.96, p.46434646, 2013.

SANTOS, G.T.; ASSIS, M.A.; GONÇALVES, G.D. et al. Determinação da digestibilidade in vitro de gramíneas do gênero Cynodon com uso de diferentes metodologias. Acta Sci., v.22, p.761-764, 2000.
SILVA, D.J.; QUEIROZ, A.C. Análise de alimentos: métodos químicos e biológicos. 3.ed. Viçosa: UFV, 2002. 235p.

TILLEY, J.M.A.; TERRY, R.A. A two-stage technique for the in vitro digestion of forage crops. J. Br. Grassl. Soc., v.18, p.104-111, 1963.

VALENTE, T.N.P.; DETMANN, E.; QUEIROZ, A.C. et al. Evaluation of ruminal degradation profiles of forages using bags made from different textiles. Rev. Bras. Zootec., v.40, p.2565-2573, 2011b.

VALENTE, T.N.P.; DETMANN, E.; VALADARES FILHO, S.C. et al. Avaliação dos teores de fibra de detergente neutro em forragens, concentrados e fezes bovinas moídas em diferentes tamanhos e em sacos de diferentes tecidos. Rev. Bras. Zootec., v.40, p.1148-1154, 2011 a. 